\title{
Erratum to: Interocular conflict attracts attention
}

\author{
Chris L. E. Paffen • Roy. S. Hessels • \\ Stefan Van der Stigchel
}

Published online: 31 March 2012

(C) Psychonomic Society, Inc. 2012
The abstract that appears below was inadvertently left out of the original article.

\begin{abstract}
During binocular rivalry, perception alternates between dissimilar images presented dichoptically. Since its discovery, researchers have debated whether the phenomenon is subject to attentional control. While it is now clear that attentional control over binocular rivalry is possible, the opposite is less evident: Is interocular conflict (i.e., the situation leading to binocular rivalry) able to attract attention? In order to answer this question, we used a change
\end{abstract}

blindness paradigm in which observers looked for salient changes in two alternating frames depicting natural scenes. Each frame contained two images: one for the left and one for the right eye. Changes occurring in a single image (monocular) were detected faster than those occurring in both images (binocular). In addition, monocular change detection was also faster than detection in fused versions of the changed and unchanged regions. These results show that interocular conflict is capable of attracting attention, since it guides visual attention toward salient changes that otherwise would remain unnoticed for longer. The results of a second experiment indicated that interocular conflict attracts attention during the first phase of presentation, a phase during which the stimulus is abnormally fused.

\footnotetext{
The online version of the original article can be found at http://dx.doi. org/10.3758/s13414-011-0256-X.

C. L. E. Paffen $(\bowtie) \cdot$ R. S. Hessels $\cdot$ S. Van der Stigchel

Helmholtz Institute and Experimental Psychology,

Utrecht University,

Heidelberglaan 2,

3584 CS Utrecht, the Netherlands

e-mail: c.l.e.paffen@uu.nl
} 